\title{
Study on the Valuation driving factors of International Oil Companies Jian Sun ${ }^{\mathrm{a},}$ * \\ ${ }^{1}$ Sinopec Research Institute of Petroleum Engineering, Beijing, China \\ aemail: sjtoefl@126.com \\ ${ }^{*}$ Corresponding author
}

Key words: International oil company; Company Value; Return on total asset; Oil price

\begin{abstract}
With the oil price varying drastically in recent years, the market value of the international oil companies changes to some extent. In order to probe the value of the companies and relevant factors, 8 famous companies listed on the Stock Exchange are taken as research objects here in. For the purpose of determining critical factors and their effect degree, the relationship between financial and operating indicators and the Management effectiveness of the company are studied with the regression analysis method. The results of the empirical work show that ROA can explain the value of the oil company effectively. In addition, oil price, company size and reputation and upstream and downstream structure are important elements by which we can know more about the value of oil companies.
\end{abstract}

\section{国际油公司价值驱动因素研究 \\ 孙键 ${ }^{a}$, \\ 中国石化石油工程技术研究院，北京，中国 \\ aemail: sjtoefl@126.com \\ *通讯作者}

关键词:国际油公司；公司价值；管理效力，油价

中文摘要:近年来随着国际油价格大幅波动, 国际油公司的市场化价值也出现了一定程度的变 化。为了确定公司的价值和其相关影响因素, 将大型国际油公司的多种财务比率同公司的价 值进行回归分析, 可确定哪些价值因素是主要影响因素及它们的相关程度。实证表明, 管理 效力在能很好的解释国际油公司的价值表现, 此外流动性、盈利能力和国际油价格也是很重 要的影响因素。

\section{1. 前言}

2005-2010年，国际石油市场价值比市场价值总体水平要高，收益能力较强，2011年下半 年这一形式逆转之下, 出现反向偏离, 即国际石油企业越来越不景气, 盈利能力大幅下挫, 很多股票价值下跌 20\%左右，国际油价格受到全球经济形势、产业升级、替代能源和环保等 因素的影响，价格大幅下跌。主要的国际油公司股票均大幅度下挫，如壳牌公司，见图1，而 同时标普500工业指数去一路上行。因此探究国际油公司真实价值及其影响因素对于我国石油 企业的发展具有重要的借鉴意义。 


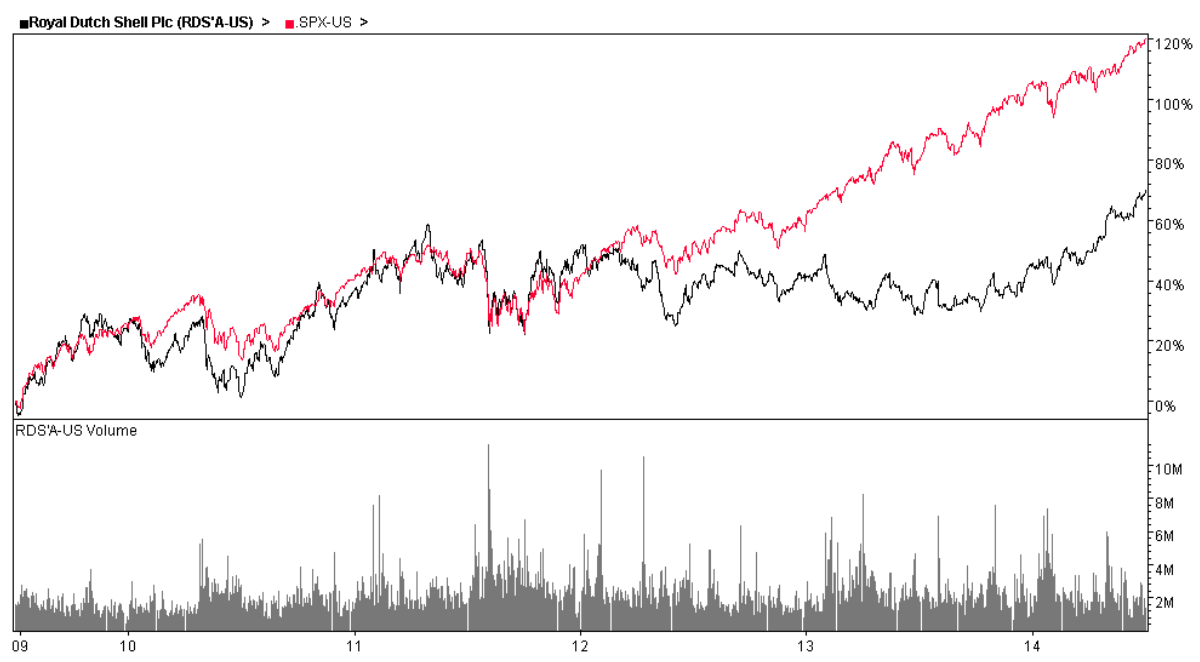

图1 壳牌公司2013-2018年股票及标准普尔500指数走势（来源：汤森路透）

\section{2. 公司价值文献综述}

国外有关公司价值研究的文献较多。如 Eugene (1970)利用美国上市公司的数据研究指出, 公司价值的影响因素有盈利能力、偿债能力、营运能力、增长、资产结构等因素 ${ }^{[1]}$ 。但是他 的研究并未考虑行业的特点。Bhattacharya (1980)等, 考虑了这一点, 对国际油公司的折旧息 税前利润(EBIDTA)、资产回报率(ROA)、净资产回报率(ROE)和营业现金流等指标进行回归 检验, 发现这些指标与公司价值的相关性较弱 ${ }^{[2]}$ 。Harold（1993）指出 EVA 与公司价值存在 高相关性 ${ }^{[3]}$ 。之后, 很多学者的研究用不同行业的公司数据均都确认了这一点, Easterbrook

（1996）在考察了市值、营业收入和息税前利润对企业价值的影响，均显著，同时发现了能 源行业中股价与收益的比值不能解释公司的价值, 因此不能作为影响公司价值的因素 ${ }^{[4]}$ 。此 外 Harris \& Raviv (1999)指出对于国际油公司, 储量和国际油价格对公司价值会产生重要的影 响 ${ }^{[5]}$ 。

Choi (2001)研究指出，国际油公司估值的特殊困难在于国际油价格测算的不合理导致会 计信息失真，从而不能准确地传递真正的经济信息 ${ }^{[6]}$; 同时由于投资者对待信息的不对称反应, 大的国际油公司在国际油价格的预计上更为保守，所以许多分析家把公司品牌作为主要参考 因素，此外他还运用1995-2000年的数据，对大型国际油公司股东财富的变化情况和折旧息税 前利润(EBIDTA)、股价与现金流的比例、权益回报率等指标进行回归检验，发现常用的财务 指标相关性很弱或者不存在, 而增长率指标和储量的相关性较高。Stuart (2004)研究了国际石 油行业盈利性和增长之间的战略困境后指出，权益回报率不能完全反映国际油公司的盈利能 力, 不适合作为衡量国际油公司业绩表现的指标，因为国际油公司具有低账面价值但仍能够 产生可观现金流的资产，如果采用权益价值计算，大多数国际油公司的权益回报率会下降 $7 \%-8 \%{ }^{[7]}$;

Gertner \& Scharfstein (2006)运用估值模型对国际油公司价值相关性进行研究，分别用净 利润和代替企业价值建立了两个模型，并通过15家最大的国际油公司2000-2005年的财务数据 和经营信息进行检验，发现传统的估值结构已不再适用 ${ }^{[8]}$ 。Benjamin（2009）对2003-2008年 美国国际油工业进行了估值方面的检验，他们对综合性国际油公司的股价与收益比和公司的 账面价值、信用、流动性杜杆、效率比率进行相关性检验，但检验结果没有反映出稳健的关 系，之后用企业价值代替账面价值之后的检验发现国际油公司未来的现金流和所探明资源量 对股价有显著的解释能力 ${ }^{[9]}$ 。此外Porta \& Sheleifer(2010)利用国际油公司的面板数据, 将国际 油公司估值和单个财务或者经营指标联系起来，选用资产回报率等指标和公司价值与现金流

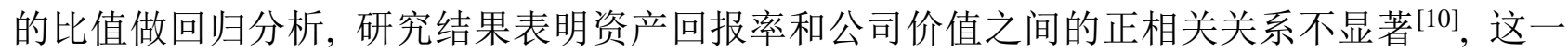
结果正好验证了Truman \& Weinstein(2012)的研究结论, 资产回报率不能反映国际油公司的价 值, 之后发现年度虚拟变量(国际油价格)和公司虚拟变量(公司品牌)反而具有很强的解释能力 
[11]。

本文试图用合并市值类参数作为公司价值衡量指标研究国际油公司价值的影响因素。同 时结合石油行业的自身特点, 试图确定在国际油价格波动的情况下, 哪些价值驱动因素对其 影响较为显著以及它们的影响程度如何，从而对国际油公司的估值特点有更深的理解。

\section{3. 研究设计}

\section{1 研究方法}

目前有多种估值方法来估计公司的市场价值,如期权定价模型、现金流贴现模型等，还有 股票分析师和专业投资者常用的乘数法。其中现金流贴现模型是最为理想的估值模型，但由 于油价波动不定，而大型国际油公司的业务往往分布在全球各地且资本总额极其庞大，所以 估计国际油企业未来的现金流量难度很大且准确性不高。在实际研究中,分析人员和投资者更 倾向于分析公司某一时期内的损益表和现金流量表中的数据,这些体现公司财务状况和盈利 能力的指标在公司的报表中都可以找到或者通过简单的计算就可以得到。苏格兰银行业在公 司估值上有相类似的观点，认为确定公司价值和流动性、盈利杜杆等之间的关系是评估公司 价值的最为重要的因素。此外，投资银行经常在其估值技术中将公司价值和单个财务指标 (如 净资产、销售收入)联系起来。

本文在总结前人模型优劣的基础上，对以上指标进行分类优选，将国际油企业流动性、 盈利能力比率、效率比率、管理效力、增长率与公司的当期价值联系起来, 建立模型探究国 际油公司价值的驱动因素。

\section{2 模型构建}

1. 模型和指标的选取

为了描述国际综合性国际油公司价值是如何与上述价值驱动因素相联系的, 本文构建模 型如下式:

$$
Y=\alpha+\beta_{1} X_{1}+\beta_{2} X_{2}+\beta_{3} X_{3}+\beta_{4} X_{5}+\beta_{5} X_{5}+\beta_{6} X_{6}+\beta_{7} X_{7}+\varepsilon
$$

其中 $\mathrm{Y}$ 代表公司价值, $\mathrm{X}_{1}$ 是企业流动性杜杆、 $\mathrm{X}_{2}$ 是盈利能力、 $\mathrm{X}_{3}$ 是效率、 $\mathrm{X}_{4}$ 是管理效力、 $\mathrm{X}_{5}$ 是增长率、 $\mathrm{X}_{6}$ 是当期国际油价格、 $\mathrm{X}_{7}$ 代表公司规模。

2. 变量定义

研究模型中各变量的计算方法如下: 债-现金。

一般学者通用的公司价值计算公式为公司价值=合并市值 + 优先股 + 少数股东权益 + 总负

流动性是财务中的重要指标, 通常包括速动比率、流动比率, 有的学者提出用现金流比 流动负债、长期负债比普通权益来表示等, 本文选用汤森路透提出的流动比率来表示流动性 杜杆，该指标在衡量资源类行业中较为稳健。流动比率=流动资产-总额/流动负债-总额。

盈利能力比率中指标较多，包括毛利率、息税折旧摊销前利润率、息税前利润率、税前 利润率和净利润率等。Gertner \& Scharfstein (2006)在其研究中指出, 在研究公司价值中, 净 利润率表现较为稳健 ${ }^{[8]}$ 。其计算公式为, 净利润率=优先股股息之前的净利润/净销售或收入 $\times 100 \%$ 。

效率指标通常按照用途分为多种，常见的有人均资产、人均销售额，应付账款周转率等, 本文选用总资产周转率这一指标，其计算公式为总资产周转率=净销售或收入/总资产。

管理效力指标本文选用众多学者和分析师非常看重的指标资产回报率，其计算公式为资 产回报率 $=($ 优先股股息前净利润 + 债务利息费用 - 资本化利息 $) \times(1$-税率 $))$ ) 上一年的总资 产 $\times 100 \%$ 。

增长率这里特指销售额增长率，其计算公式为=:1-(上期销售收入/当期销售收入) $\times 100 \%$ 。 国际油价格选用澳洲纽卡斯尔港动力油价。公司规模选用公司当期的营业收入来表示。 


\section{3 样本选取}

本文所选取的样本是在纽约证券交易所(NYSE)上市的国际综合性国际油公司, 得到了以 下8家: EXXON、SHELL、TOTAL、CHEVRON、BP、Conoco Phillip、PETROBRAS 和STATOIL。 这些国际油公司是目前全球最大的综合性国际油公司, 比较具有代表性和研究意义。所采用 的数据均来自汤森路透 $\mathrm{T} 1$ 数据库中2009-2013年面板数据, 本研究共得到 246 个会计年度数据。 统计软件使用SPSS16.0。

\section{4. 实证分析}

\section{1 变量相关性分析}

从表1中可以发现大部分变量之间的相关系数很低,说明大多数变量之间不存在多重共线 的情况,可以独立地代表其各自的经济含义。但油价和公司规模的的相关程度很高,相关系数为 0.754 ,石油作为重要的大宗商品和战略能源,和市场总体的运行情况是息息相关的, 油价高, 公司营业收入必然升高。鉴于它们的相关程度很高，笔者只选取油价加入到回归模型。

表1 变量间的皮尔森相关系数

\begin{tabular}{cccccccc}
\hline & 流动性杜杆 & 盈利能力 & 效率 & 管理效力 & 增长率 & 国际油价格 & 公司规模 \\
流动性杜杆 & 1 & & & & & & \\
盈利能力 & 0.01 & 1 & & & & & \\
效率 & 0.13 & 0.23 & 1 & & & & \\
管理效力 & 0.04 & 0.45 & 0.12 & 1 & & & \\
增长率 & -0.01 & 0.82 & 0.06 & -0.267 & 1 & & \\
国际油价格 & 0.003 & 0.047 & 0.014 & 0.014 & -0.241 & 1 & 1 \\
公司规模 & 0.04 & 0.21 & 0.156 & 0.324 & 0.323 & 0.754 & 1 \\
\hline
\end{tabular}

\section{2回归分析}

下面笔者将以上指标加入模型，试图寻找国际油公司估值中的价值驱动因素。回归结果 见表2。

表2 多变量模型回归结果

\begin{tabular}{ccc}
\hline 变量 & 系数 & $\mathrm{T}$ 检验 \\
\hline $\mathrm{C}$ & 0.403 & 3.268 \\
流动性 & 0.003 & 1.836 \\
盈利能力 & $0.540^{* *}$ & 1.621 \\
效率 & $0.230^{* *}$ & 0.903 \\
管理效力 & $3.630 * * *$ & 3.620 \\
增长率 & 0.042 & 0.857 \\
当期国际油价格 & $0.002^{*}$ & 1.956 \\
调整 $\mathrm{R}^{2}$ & 0.635 & \\
$\mathrm{~F}$ 检验 & 27.306 &
\end{tabular}

注: *** 表示变量通过了显著水平为 $1 \%$ 的统计检验。**表示变量通过了显著水平为 $5 \%$ 的统计检验, * 表示变 量通过了显著水平为 $10 \%$ 的统计检验。

从表 2 的回归结果可以看出，管理效力通过了显著水平 $1 \%$ 的 $\mathrm{T}$ 检验，同时油价通过了显著 性为 $10 \%$ 的 $\mathrm{T}$ 统计检验, 流动性和盈利能力通过了显著性为 $5 \%$ 的 $\mathrm{T}$ 统计检验, 这说明这些变量 对于国际油公司价值的影响还是十分显著的。其中盈利能力对国际油公司价值的促进作用是 可以预料的,稳定的经营状况、具有竞争力的盈利能力有利于公司价值的形成。而效率和增长 率两个变量没有通过检验，对国际油公司价值的影响不显著。

流动性指标显著，说明流动资产之中现金、有价证券、应收账款有影响，但是对于国际 石油企业来讲存货和摊销等变现能力也应该算在效率中，这可能是该指标不显著的原因。 
公司盈利能力比重与公司价值存在正相关关系，只有高盈利能力的企业才能通过持续经 营活动为其股东创造价值。

效率指标显著，效率与企业价值存在倒U型关系，即存在一个拐点，任何资产都是一类生 产要素, 某一生产要素都存在最佳的比例, 过高过低都会损害企业资产生产效率, 从而损害 企业价值。

管理效力指标通过了显著性为 $1 \%$ 的统计 $\mathrm{T}$ 检验，这说明了它和国际油公司价值的正相关 关系是十分显著的，运用资产获利能力强的国际油公司其市场价值也相应更高。

销售额增长率在解释公司价值方面并不显著,这是因为增长只是表象，支撑其长期持续增 长的则是其盈利能力; 盈利能力低下的企业不可能实现长期、持续的高增长, 即使通过股权 融资短期内实现较高增长，也因为其资金使用效率低下、仍然是价值毁灭者，只有高盈利能 力的企业才能通过持续经营活动为其股东创造价值, 也只有基于高盈利能力的增长对于其股 东才有价值。

油价和综合性国际油公司价值的正相关关系,究其原因,笔者认为油价下跌会使整个国际 石油行业的价值减少。另一方面，油价上升而短时间内国际油能源的不可替代性导致投资者 和消费者相信油价还会走高，而国际油公司的核心资产就是石油资源，其价值必然会大幅提 高。

\section{5. 结论}

本文在总结前人研究的基础上，优选公司关键的一些财务指标，将国际油公司的价值联 系起来, 试图从回归分析中寻找出主要相关信息, 以确定国际油公司的价值影响因素, 为以后 以这些指标为基础从实务中评估国际油公司的价值做好准备。研究结果表明，在评估大型综 合性国际油公司价值时,管理效力能有效地反映国际油公司的市场价值。此外，国际油公司效 率指标、盈利能力和油价也和国际油公司的价值显著正相关。总之, 本文的研究结论对于评 估国际油公司价值以及为油公司决策者提供经营决策都有很好的帮助。

\section{References}

[1] Fama, Eugene F. 1970, Effective Capital Markets: A Review of Theory and Empirical Work J. Finance, 25 (May):383-417.

[2] Bhattacharya, S., 1980, Nondissipative signaling structures and dividend policy, Quarterly Journal of Economics, vol.95, 1-14.

[3] Demsetz, Harold, 1993, The structure of ownership and the theory of the firm, Journal of Law and Economics, vol.26,375-390.

[4] Easterbrook, F. H., 1996, Two agency costs explanation of dividends, American Economic Review, vol.74, 650-659.

[5] Harris, M. and Raviv, 1999, A sequential signaling model of convertible debt policy, Journal of Finance, vol.40, 1263-1281.

[6] Choi, D., 2001, Stock repurchase under informational asymmetry: The empirical evidence, Economic forces and the stock market. Journal of Business, vol.69, 383-403.

[7] Gilson, Stuart, 2004, Management turnover and financial distress, Journal of Financial Economics, vol. 25, 241-262.

[8] Asquith, P., R. Gertner, and D.Scharfstein, 2006, An anatomy of financial distress: An examination of junk-bond issuers, NBER working paper no. 3942 (NBER, Cambridge, MA)

[9] Clark, Truman A. and Mark Weinstein, 2012, The behavior of the common stock of bankrupt firms, Journal of finance, vol.38, 489-450. 